\title{
1 Anemia amelioration by lactose infusion during trypanosomosis could be associated with
}

\section{2 erythrocytes membrane de-galactosylation}

3 E.O. Balogun ${ }^{\text {a,b,c, }}$, J.A.B. Balogun ${ }^{\mathrm{d}}$, S. Yusuf ${ }^{\mathrm{e}}$, H.M. Inuwa ${ }^{\mathrm{a}}$, I.S. Ndams ${ }^{\mathrm{f}}$, P. Sheridan ${ }^{\mathrm{g}}$, D.K.

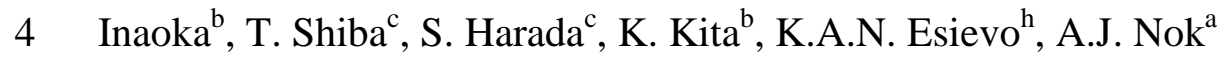

$5 \quad{ }^{\mathrm{a}}$ Department of Biochemistry, ${ }^{\mathrm{f}}$ Department of Biological Sciences, and ${ }^{\mathrm{h}}$ Department of

6 Veterinary Pathology and Microbiology, Ahmadu Bello University, Zaria 2222, Nigeria.

$7 \quad{ }^{b}$ Department of Biomedical Chemistry, Graduate School of Medicine, The University of Tokyo,

8 7-3-1 Hongo, Bunkyo-ku, Tokyo 113-0033, Japan.

$9 \quad{ }^{\mathrm{c}}$ Department of Applied Biology, Graduate School of Science and Technology, Kyoto Institute

10 of Technology, Sakyo-ku, Kyoto 606-8585, Japan.

$11{ }^{\mathrm{d}}$ Department of Biological Sciences, Federal University Dutse, P.M.B.1756, Dutse, Jigawa state, 12 Nigeria.

$13{ }^{\mathrm{e}}$ Department of Physiology, Faculty of Biomedical Sciences, Kampala International University, 14 Uganda.

$15{ }^{\mathrm{g}}$ Laboratory of DNA Information Analysis, Human Genome Center, Institute of Medical

16 Science, The University of Tokyo, 4-6-1 Shirokanedai, Minato-ku, Tokyo 108-8639, Japan.

17 Keywords: Trypanosomosis, Anemia, Sialidase, RBC membrane galactose

$18 \quad$ * Corresponding author: $\quad$ E-mail address: balogun1@m.u-tokyo.ac.jp (EO. Balogun)

Department of Biomedical Chemistry, 


\section{ABSTRACT}

25 African trypanosomosis is a potentially fatal disease that is caused by extracellular parasitic

26 protists known as African trypanosomes. These parasites inhabit the blood stream of their

27 mammalian hosts and produce a number of pathological features, amongst which is anemia.

28 Aetiology of the anemia has been partly attributed to an autoimmunity-like mediated

29 erythrophagocytosis of de-sialylated red blood cells (dsRBCs) by macrophages. Lactose infusion

30 to infected animals has proven effective at delaying progression of the anemia. However, the

31 mechanism of this anemia prevention is yet to be well characterized. Here, the hypothesis of a

32 likely induced further modification of the dsRBCs was investigated. RBC membrane galactose

33 (RBC m-GAL) and packed cell volume (PCV) were measured during the course of experimental

34 trypanosomosis in mice infected with Trypanosoma congolense (stb 212). Intriguingly, while the

35 membrane galactose on the RBCs of infected and lactose-treated mice (group D) decreased as a

36 function of parasitemia, that of the lactose-untreated infected group (group C) remained

37 relatively constant, as was recorded for the uninfected lactose-treated control (group B) animals.

38 At the peak of infection, the respective cumulative percent decrease in PCV and membrane

39 galactose were 30 and 185 for group D, and 84 and 13 for group C. From this observed inverse

40 relationship between $\mathrm{RBCs}$ membrane galactose and PCV, it is logical to rationalize that the

41 delay of anemia progression during trypanosomosis produced by lactose might have resulted

42 from an induction of galactose depletion from dsRBCs, thereby preventing their recognition by

43 the macrophages. 


\section{Introduction}

Trypanosomes are blood parasites and the causative agents of protozoan diseases known as nagana in animals, sleeping sickness and Chagas' disease in humans. In addition to

47 Trypanosoma brucei, other causes of nagana include Trypanosoma congolense and

48 Trypanosoma vivax, which are all transmitted to the mammalian host by a parasite-carrying

49 tsetse during its blood meal (Jamonneau et al 2004; Krafsur, 2009). Sleeping sickness and

50 nagana are a major health problem in sub-Saharan Africa, and the economic impact of the animal

51 disease is a severe constraint against the development of the region (Fenwick et al., 2005).

52 Anemia has long been established as a prominent pathological feature during

53 trypanosomosis (Woodruff et al., 1973; Jennings et al., 1974; Salgado et al., 2011). It is largely

54 attributed to the rapid loss of red blood cells (RBCs) from circulation, rather than a defective 55 erythropoiesis (Biryomumaisho and Katunguka-Rwakishaya, 2007). A number of hypotheses

56 have been put forward to explain the precise mechanism of this increased erythrocytes

57 loss/destruction. They include: immuno-destruction, resulting from adsorption of parasites'

58 antigenic material on to RBCs membranes (Woo and Kobayashi, 1975); membrane-lipid

59 peroxidation, leading to oxidative damage of the RBCs (Igbokwe et al., 1996; Wolkmer et al.,

60 2009); and phagocytosis of de-sialylated RBCs (Esievo et al., 1982). The last mechanism is the

61 best-understood and widely accepted cause of the anemia (Guegan et al., 2013).

The erythrocyte membrane of human and animal species is heavily sialylated (Yaari,

63 1969; Mehdi et al., 2012), with the sialic acids (SAs) occupying terminal positions and masking

64 an underlying galactosyl residue of cell membrane sialoglycoproteins (Varki, 2001). Membrane

65 SAs have been shown to be a critical anti-recognition molecule, protecting RBCs from

66 phagocytosis (Lee et al., 1988; Bratosin et al., 1998) by cells of the mononuclear phagocyte 
67 system (MPS). Removal of SAs usually by actions of sialidases results in exposing the

68 previously masked penultimate galactosyl residue on RBC membrane, which becomes

69 recognisable by the galactose specific lectins on macrophages, leading to

70 erythrophagositosis/sequestration of the asialo-RBCs, hence, the anemia (Brastosin et al., 1998;

71 Guegan et al., 2013). Sialidases have been reported in different species of trypanosomes; their

72 hydrolytic capacity on RBC membrane SAs, and the role in anemia pathogenesis are well studied

73 (Nok and Balogun, 2003; Nok et al., 2003; Buratai et al., 2006; Coustou et al., 2012). As a

74 result, the use of sialidase inhibitors has been thought to hold great prospect for chemotherapy or

75 at least anemia prevention during trypanosomosis (Antoine-Moussiaux et al., 2009). However,

76 there is yet to be any successful effort towards treatment of trypanosome-infected animals with

77 sialidase inhibitors. The only effort in this direction is focused on the use of lactose infusion to

78 ameliorate the anemia.

Although, the amelioration of anemia by lactose infusion during trypanosomosis is

80 documented (Umar et al., 1998: Fatihu et al., 2008), there is a dearth of information on this, and

81 the mechanism of the slow anemia progression remains inconclusive. To date, the only suggested

82 mechanism is a competition for the galactose-specific lectins on MPS by lactose. However, due

83 to the abundance of RBCs with exposed galactose during the disease as a result of the high

84 parasite sialidase activity, it is logical to suppose that competition alone, between infused lactose

85 and the circulating asialo-RBCs cannot account for such magnitude and efficiency of RBCs'

86 protection from MPS. This has led to a speculation for the existence of additional mechanism(s)

87 of anemia prevention by lactose infusion during trypanosomosis. In the present study, the

88 presence of a potential glycoconjugate-modifying enzyme during lactose infusion to

89 trypanosome-infected mice was examined. We previously reported an elevated serum 
90 glycosidase activity in the lactose-infused animals (Nok and Balogun, 2003), and herein, for the

91 first time discuss its possible role in further modification of desialylated RBCs as a possible

92 mechanism of asialo-RBCs' protection from recognition and sequestration by the MPS.

93

\section{2. Materials and methods}

95 Same-colony 5-6 weeks old female BALB/c mice, weighing 20-22 g, were procured

96 from the animal unit of Faculty of Pharmaceutical Sciences, Ahmadu Bello University

97 Zaria-Nigeria. They were maintained on commercial pellet diet and water ad libitum under

98 standard housing conditions. All animals used in this work were treated in accordance with the

99 approved guidelines for animal experiments as reviewed by Ahmadu Bello University animal

100 care and use committee. The animals were randomly separated to four groups (A-D) of four mice

101 each. Starting from day 0; group A were not infused and not infected, group B were infused but

102 not infected, group C were not infused but infected, and group D were infused and infected.

103 Infection was by a single intraperitoneal inoculation with $10^{4}$ Trypanosoma congolense (stb 212)

104 cells, while infusion was twice-a-day intraperitoneal administration of lactose in phosphate

105 buffered saline (PBS) solution at a dosage of $0.5 \mathrm{~g}$ per $\mathrm{Kg}$ body weight throughout the animal

106 survival period.

107 Parasitemia was monitored daily by microscopic examination of thin blood smears, and

108 in order to evaluate the loss of RBC that is often associated with the infection, microhematocrit

109 method was used to monitor packed cell volume (PCV) at two days interval. To examine the

110 relationship between PCV profiles and RBC surface modifications in trypanosome-infected

111 animals and to investigate if the modifications were affected by lactose infusion, RBC ghosts

112 were prepared as described in Natala et al. (2013), and analysed. We have previously reported a 
113 correlation between parasitemia and trypanosome sialidase activity with RBC desialylation and 114 decreased PCV in T. congolense-infected animals (Nok and Balogun, 2003). To investigate the

115 possible further modifications occurring on RBCs during infection, susceptibility of the exposed

116 galactosyl residues on membranes of de-sialylated RBCs to the released glycosidase was

117 evaluated. A modified method of Stibler et al. (1983) was used to quantify RBC membrane

118 galactose. Briefly, protein concentration of homogenous suspension of prepared RBC

119 membranes of all mice groups (A-D) was determined according to Lowry et al., (1951).

120 Normalized by constant protein amount $(0.3 \mathrm{~g} / \mathrm{l})$, triplicate samples of the RBC membranes were

121 hydrolysed in $200 \mu \mathrm{l}$ reaction containing $0.1 \mathrm{M} \mathrm{HCl}$ at $95^{\circ} \mathrm{C}$ for $20 \mathrm{~h}$. Mineral oil was overlayed

122 on each reaction to prevent evaporation. The subsequent steps were as described in Stibler et al.

123 (1983) except that amounts of NAD and D-galactose dehydrogenase per reaction were reduced

124 to $100 \mathrm{nmol}$ and $50 \mathrm{mU}$, respectively. RBC membrane SAs (RBC m-SAs) was quantified as

125 described in Nok and Balogun (2003), using the prepared ghost cells. Two-tailed independent

126 t-test was used to analyse the differences in PCV, RBC m-SAs, and RBC m-GAL between the

127 groups.

128 


\section{Results and Discussion}

The rate of parasitemia development varied in all the infected mice but all had above

$13110^{6}$ parasites/mL of blood by day 5 post-infection. The parasitemia progressed, demonstrating

132 the classical waves of infection (data not shown). From our previous observation, significant

133 number of mice died during the fourth wave, therefore, the peak of infection was taken as a level

134 of approximately $10^{8}$ parasites $/ \mathrm{mL}$ that was attained at the third parasitemic bout.

135 Similar to our previous report (Nok and Balogun, 2003) and that of Fatihu et al., (2008),

136 lactose infusion to the infected animals significantly maintained PCV by a value of over $10 \%$

137 above the non-lactose treated infected group, even at peak of parasitemia (Fig. 1). Surprisingly,

138 this difference in PCV could not be accounted for by the difference in RBC membrane sialic

139 acids (RBC m-SAs), considering that the values were comparable for the lactose-treated and

140 untreated infected groups (Table 1). These values were 60\% of that for uninfected lactose-treated

141 and untreated control groups (Table 1), implying that terminal RBC m-SAs were cleaved by

142 trypanosome sialidase even in the lactose-treated animals, hence the erythrocytes protection was

143 not because dsRBCs were not produced, but for other reason(s). However, in contrast to the

144 similar RBC m-SAs in the infected mice, there was a coincidental significant depletion of RBC

145 membrane galactose (RBC m-GAL), to a difference of about $-30 \%$ in the lactose-treated group

146 with reference to the untreated group. The RBC m-GAL of the untreated group was similar to the

147 uninfected controls (Table 1).

148 To further elucidate the variations in RBC m-GAL at the different stages of parasitemia,

149 the RBC ghosts of the animals were analysed. Results revealed that while the RBC m-GAL value

150 remained fairly constant through the course of infection in the untreated group, it began to get

151 depleted when trypanosome number reached $10^{4}$ parasites/mL (Fig 2). The depletion continued 
152 and was negatively correlated $(\mathrm{r}=-0.73, \mathrm{P}<0.003)$ to parasitemia level (Fig 2$)$, resulting in

153 about $-180 \%$ relative cumulative change. However, the depletion was no longer proportional to

154 trypanosome load beyond $10^{7}$ parasites $/ \mathrm{mL}$, as seen from the relative similarity in the value of

$155 \mathrm{RBC}$ m-GAL at parasitemia level between $10^{7}$ and $10^{8}$ parasites $/ \mathrm{mL}$ (Fig 2). This result suggests

156 that the infusion of lactose to the infected mice led to an accelerated depletion of galactose from

157 the membrane of RBCs.

158 In order to obtain a meaningful interpretation of the relationship between RBC m-GAL

159 and PCV at the different parasitemia level, these data for the lactose-treated and untreated groups

160 were plotted, subjected to linear regression analysis, and compared. The regression equation for

161 treated group is given by the formula $\mathrm{PCV}=-2.196 \log _{10}(N)+56.28(\mathrm{P}=0.005)$ and that for the

162 untreated group as $\mathrm{PCV}=-4.366 \log _{10}(N)+57.55(\mathrm{P}<0.001)$, where $N$ is the parasite number per

$163 \mathrm{~mL}$ (Fig. 3a and b). A visual inspection of the plotted data points clearly reveals that PCV

164 percent decreases as the parasitemia level increases in both cases. The regression equations

165 quantify these observed relationships. Values of the regression coefficients; -2.1964 for the

166 lactose-treated and -4.3661 for the untreated animals, imply that the rate of drop in PCV in the

167 untreated group is twice that for the lactose-treated animals. Conversely, judging from the RBC

168 m-GAL curves and their regression coefficients; -0.1313 for the lactose-treated and -0.0045 for

169 the untreated (Fig 3a and b), there was no significant change in the RBC m-GAL values (P >

1700.25 ) for the untreated group, while that for treated animals dropped considerably (at a rate

171 approximately 30 times higher) as parasitemia increased $(\mathrm{P}<0.001)$. These findings suggest that

172 the relative maintenance of PCV in the lactose-treated animals is accompanied by a sharp

173 decrease in the amount of their erythrocytes membrane galactose. 
175 trypanosome-infected animals that receive lactose treatment has revealed an interesting

176 phenomenon that may help to provide an insight into the mechanism of anemia amelioration.

177 From the aforementioned, coupled with our previous observation that the serum of this group of 178 animals possessed an increased ability to cleave galactose from an artificial substrate,

179 4-Methylumbelliferyl galactose (Nok and Balogun, 2003); we can infer that lactose infusion 180 resulted in an increased amount of a secreted galactose hydrolyzing enzyme. Moreover, a 181 separate group reported that Trypanosoma evansi contained beta galactosidase (Sallau et al., 182 2008). Although we could not locate any open reading frame (ORF) for galactosidase gene in the 183 genome of $T$. congolense, four putative glucosidase ORFs were identified from the TritrypDB 184 (http://tritrypdb.org/tritrypdb/). This could mean that at least one of the genes' products is a 185 non-specific glucosidase that also cleaves galactose, hence; we refer to it broadly as a 186 glycosidase. It is logical to therefore rationalize that elevation of the glycosidase in the 187 lactose-treated infected animals modified the surface of the dsRBCs further by removing the 188 exposed galactose, making them unrecognizable by the galactose-specific lectins on 189 macrophages, hence reducing their rates of removal from circulation and leading to a reduction 190 in the rate of anemia progression. On the contrary, there was probably low plasma glycosidase in 191 the lactose-untreated animals (as suggested by higher galactose content of their dsRBCs); 192 resulting in an unchanged surface (exposed galactose) of the dsRBCs, which were rapidly 193 recognized and sequestered by the immune cells-erythrophagosytosis. To conclude, our present 194 observation revealed a likely mechanism of anemia suppression by lactose infusion to 195 trypanosome-infected animals. Lactose induced the cleavage of exposed galactose on the 196 membrane surface dsRBCs that are produced by the trypanosomal sialidases, making them 
197 unrecognisable by the galactose-specific lectins of macrophages, and preventing their

198 sequestration by these immune cells, thus, reducing the rate of anemia progression.

199

200 Acknowledgements

Authors thank Balogun Olukunmi (Graduate School of Medicine, The University of

202 Tokyo) for critical reading of the manuscript and language corrections. This work was partly

203 supported by a grant from Ahmadu Bello University Board of Research (ABUBR). EOB is

204 presently a postdoctoral fellow of the Japan Society for the Promotion of Science (JSPS).

205

206 


\section{References}

208 Antoine-Moussiaux, N., Buscher, P., Desmecht, D., 2009, Host-parasite interactions in trypanosomiasis: on the way to an antidisease strategy. Infect. Immun. 77, 1276-1284.

210 Biryomumaisho, S., Katunguka-Rwakishaya, E., 2007, The pathogenesis of anaemia in goats experimentally infected with Trypanosoma congolense or Trypanosoma brucei: use of the myeloid:erythroid ratio. Vet. Parasitol. 143, 354-357.

Bratosin, D., Mazurier, J., Tissier, J.P., Estaquier, J., Huart, J.J., Ameisen, J.C., erythrocyte phagocytosis by macrophages. A review. Biochimie 80, 173-195.

Buratai, L.B., Nok, A.J., Ibrahim, S., Umar, I.A., Esievo, K.A., 2006, Characterization of sialidase from bloodstream forms of Trypanosoma vivax. Cell Biochem. Funct. 24, $71-77$.

Coustou, V., Plazolles, N., Guegan, F., Baltz, T., 2012, Sialidases play a key role in infection and anaemia in Trypanosoma congolense animal trypanosomiasis. Cell Microbiol. 14, 431-445.

Esievo, K.A., Saror, D.I., Ilemobade, A.A., Hallaway, M.H., 1982, Variation in

Fatihu, M.Y., Adamu, S., Umar, I.A., Ibrahim, N.D., Eduvie, L.O., Esievo, K.A., 2008, cattle. 2. Packed cell volume. Onderstepoort J. Vet. Res. 75, 181-187. erythrocyte surface and free serum sialic acid concentrations during experimental Trypanosoma vivax infection in cattle. Res. Vet. Sci. 32, 1-5. 
Guegan, F., Plazolles, N., Baltz, T., Coustou, V., 2013, Erythrophagocytosis of desialylated red blood cells is responsible for anaemia during Trypanosoma vivax infection. Cell Microbiol. 15, 1285-303

233

Igbokwe, I.O., Umar, I.A., Omage, J.J., Ibrahim, N.D., Kadima, K.B., Obagaiye, O.K., Saror, D.I., Esievo, K.A., 1996, Effect of acute Trypanosoma vivax infection on cattle erythrocyte glutathione and susceptibility to in vitro peroxidation. Vet. Parasitol. 63, 215-224.

Jamonneau, V., Ravel, S., Koffi, M., Kaba, D., Zeze, D.G., Ndri, L., Sane, B., Coulibaly, B., Cuny, G., Solano, P., 2004, Mixed infections of trypanosomes in tsetse and pigs and their epidemiological significance in a sleeping sickness focus of Cote d'Ivoire. Parasitology 129, 693-702.

Jennings, F.W., Murray, P.K., Murray, M., Urquhart, G.M., 1974, Anaemia in trypanosomiasis: studies in rats and mice infected with Trypanosoma brucei. Res. Vet. Sci. $16,70-76$.

Krafsur, E.S., 2009, Tsetse flies: genetics, evolution, and role as vectors. Infect. Genet. Evol. 9, 124-141.

Lee, H., Kelm, S., Yoshino, T., Schauer, R., 1988, Carbohydrate specificity of the galactose-recognizing receptor of rat peritoneal macrophages. Biol. Chem. Hoppe Seyler 369, 705-714.

Lowry, O.H., Rosebrough, N.J., Farr, A.L., Randall, R.J., 1951, Protein measurement with the Folin phenol reagent. J. Biol. Chem. 193, 265-275.

Mehdi, M.M., Singh, P., Rizvi, S.I., 2012, Erythrocyte sialic acid content during aging in humans: correlation with markers of oxidative stress. Dis. Markers 32, 179-186. 
253 Natala, A.J., Balogun, E.O., Balogun, J.A., Inuwa, H.M., Nok, A.J., Shiba, T., Harada,

254 S., Kita, K., Agbede, R.I., Esievo, K.A., 2013, Identification and characterization of sialidase-like activity in the developmental stages of Amblyomma variegatum. J. Med. Entomol. 50, 85-93.

Nok, A.J., Balogun, E.O., 2003, A bloodstream Trypanosoma congolense sialidase

Nok, A.J., Nzelibe, H.C., Yako, S.K., 2003, Trypanosoma evansi sialidase: surface could be involved in anemia during experimental trypanosomiasis. J. Biochem.

$133,725-730$.
localization, properties and hydrolysis of ghost red blood cells and brain cells-implications in trypanosomiasis. Z. Naturforsch C 58, 594-601.

Salgado, B.S., Battaglia, C.T., Stuchi, R.S., Cadioli, F.A., Rozza, D.B., Machado, G.F., 2011, What is your diagnosis? Lymphadenopathy in a cow with severe anemia. Bovine trypanosomiasis. Vet. Clin. Pathol. 40, 103-104.

Sallau, A.B., Ibrahim, M.A., Salihu, A., and Yusuf, I.A., 2008, Bloodstream form of Trypanosoma evansi contains $\beta$-galactosidase. Middle-East J. Sci. Res. 3, 49-52.

Stibler, H., Sydow, O., 1983, The sialic acid and galactose concentrations in erythrocyte membranes in patients with myotonic dystrophy, limb-girdle and facioscapulohumeral dystrophy. J. Neurol. Sci. 59, 389-399.

Umar, I.A., Omage, J.J., Shugaba, A., Igbokwe, I.O., Ibrahim, N.D., Kadima, K.B.,

275 Varki, A., 2001, Analysis of oligosaccharide negative charge by anion-exchange 
277 Wolkmer, P., da Silva, A.S., Traesel, C.K., Paim, F.C., Cargnelutti, J.F., Pagnoncelli,

278 M., Picada, M.E., Monteiro, S.G., Lopes, S.T., 2009, Lipid peroxidation associated with

279 anemia in rats experimentally infected with Trypanosoma evansi. Vet. Parasitol. 165,

$280 \quad 41-46$.

281 Woo, P.T., Kobayashi, A., 1975, Studies on the anaemia in experimental African trypanosomiasis. 1. A preliminary communication on the mechanism of the anaemia. Ann. Soc. Belg. Med. Trop. 55, 37-45.

284 Woodruff, A.W., Ziegler, J.L., Hathaway, A., Gwata, T., 1973, Anaemia in African trypanosomiasis and 'big spleen disease' in Uganda. Trans. R. Soc. Trop. Med. Hyg. 67, 329-337.

287 Yaari, A., 1969, Mobility of human red blood cells of different age groups in an electric field. Blood 33, 159-163. 
Table and Figure captions

291 Table 1. Erythrocytes membrane galactose (RBC m-GAL) and sialic acids (RBC m-SAs).

292 Results are mean \pm standard deviation (SD). Different letters in the same column indicate a

293 significance difference $(\mathrm{P}<0.05)$.

295 Fig. 1. Effect of lactose infusion on packed cell volume (PCV) of Trypanosoma congolense

296 infected mice (four per group). Bars represent averages of two readings at peak of parasitemia,

297 while error bars are \pm SD. Different letters indicate a significance difference $(\mathrm{P}<0.05)$.

299 Fig. 2. The variation of RBC m-GAL at different level of parasitemia. Galactose was

300 quantified in erythrocytes ghosts prepared from blood of Trypanosoma congolense infected

301 mice that were treated (group D; solid line) and untreated (group C; dotted line) with lactose.

302 Plots were prepared using averages of three determinations, and error bars represent \pm SD.

304 Fig. 3. Comparison between the variations of RBC m-GAL (diamond lines) and PCV (cube 305 lines) induced by lactose infusion to Trypanosoma congolense infected mice. (A) is lactose 306 treated group (D), while (B) is the untreated group (C). The respective regression lines are shown 307 as dotted and solid lines. The generated regression equations suggest that maintenance of PCV in 308 group D mice is attributable to RBC m-GAL depletion from the membrane of dsRBCs. Each plot 309 is an average of three experiments, but for the purpose of clarity, \pm SD were not indicated. 
Table 1: Erythrocytes membrane galactose (RBC m-GAL) and sialic acids (RBC m-SAs).

\begin{tabular}{llll}
\hline Animal groups & $\begin{array}{l}\text { Protein } \\
(\mathrm{g} / \mathrm{L})\end{array}$ & $\begin{array}{l}\text { RBC m-GAL } \\
(\mathrm{mmol} / \mathrm{g} \text { Prot })\end{array}$ & $\begin{array}{l}\text { RBC m-SAs } \\
(\mu \mathrm{mol} / \mathrm{g} \text { Prot })\end{array}$ \\
\hline Not infected, not lactose-treated (A) & $28.8 \pm 0.5$ & $1.37 \pm 0.05^{\mathrm{a}}$ & $81.5 \pm 1.8^{\mathrm{c}}$ \\
Not infected, lactose-treated (B) & $25.9 \pm 0.6$ & $1.38 \pm 0.05^{\mathrm{a}}$ & $80.9 \pm 2.1^{\mathrm{c}}$ \\
Infected, not lactose-treated (C) & $28.6 \pm 0.8$ & $1.35 \pm 0.06^{\mathrm{a}}$ & $49.5 \pm 1.9^{\mathrm{d}}$ \\
Infected, lactose-treated (D) & $26.3 \pm 0.6$ & $0.65 \pm 0.06^{\mathrm{b}}$ & $48.1 \pm 1.9^{\mathrm{d}}$ \\
\hline
\end{tabular}

RBC m-GAL: red blood cells membrane galactose; RBC m-SAs: red blood cells membrane sialic acids 


\section{Balogun et al}

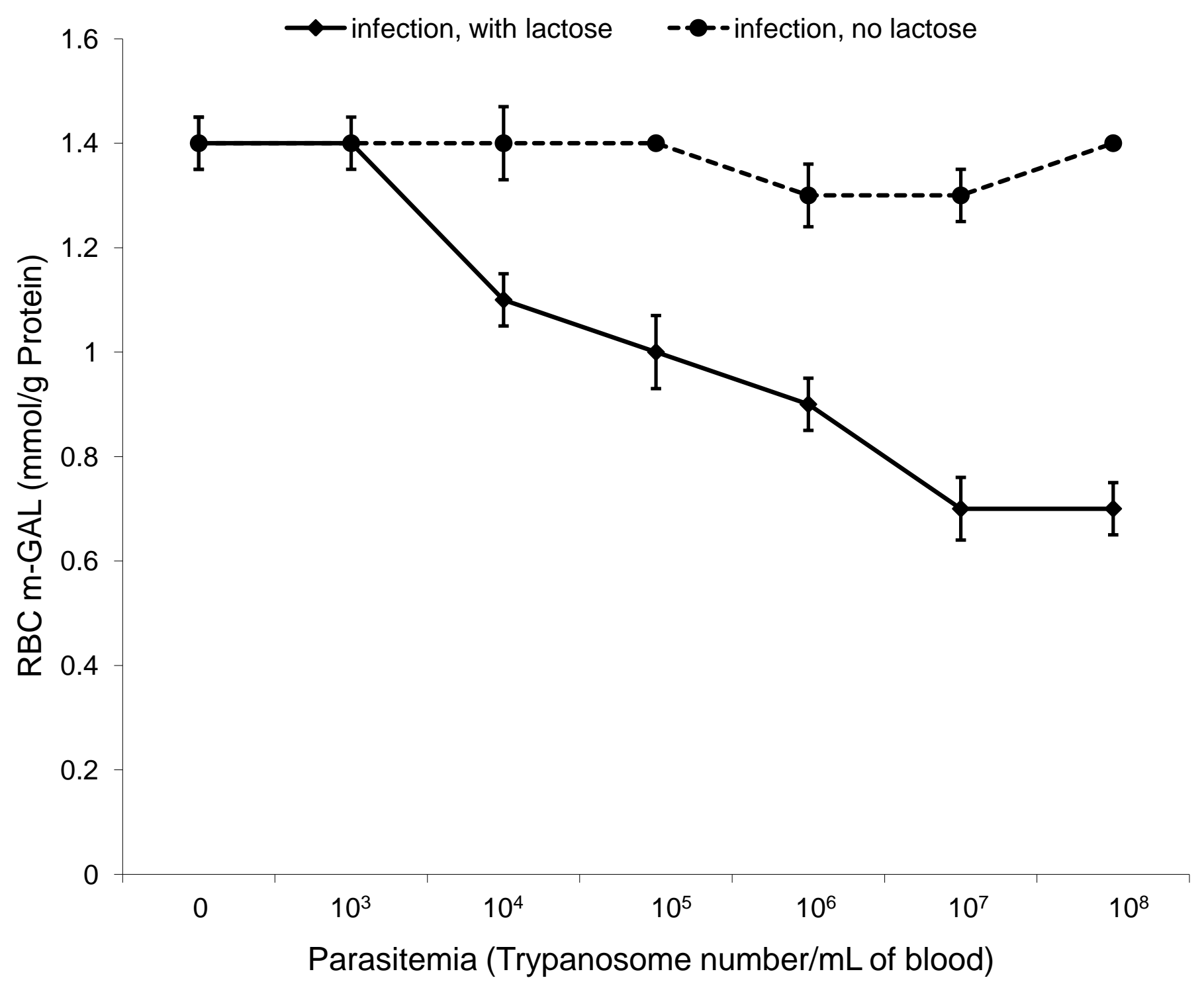

Fig. 2 
Balogun et al

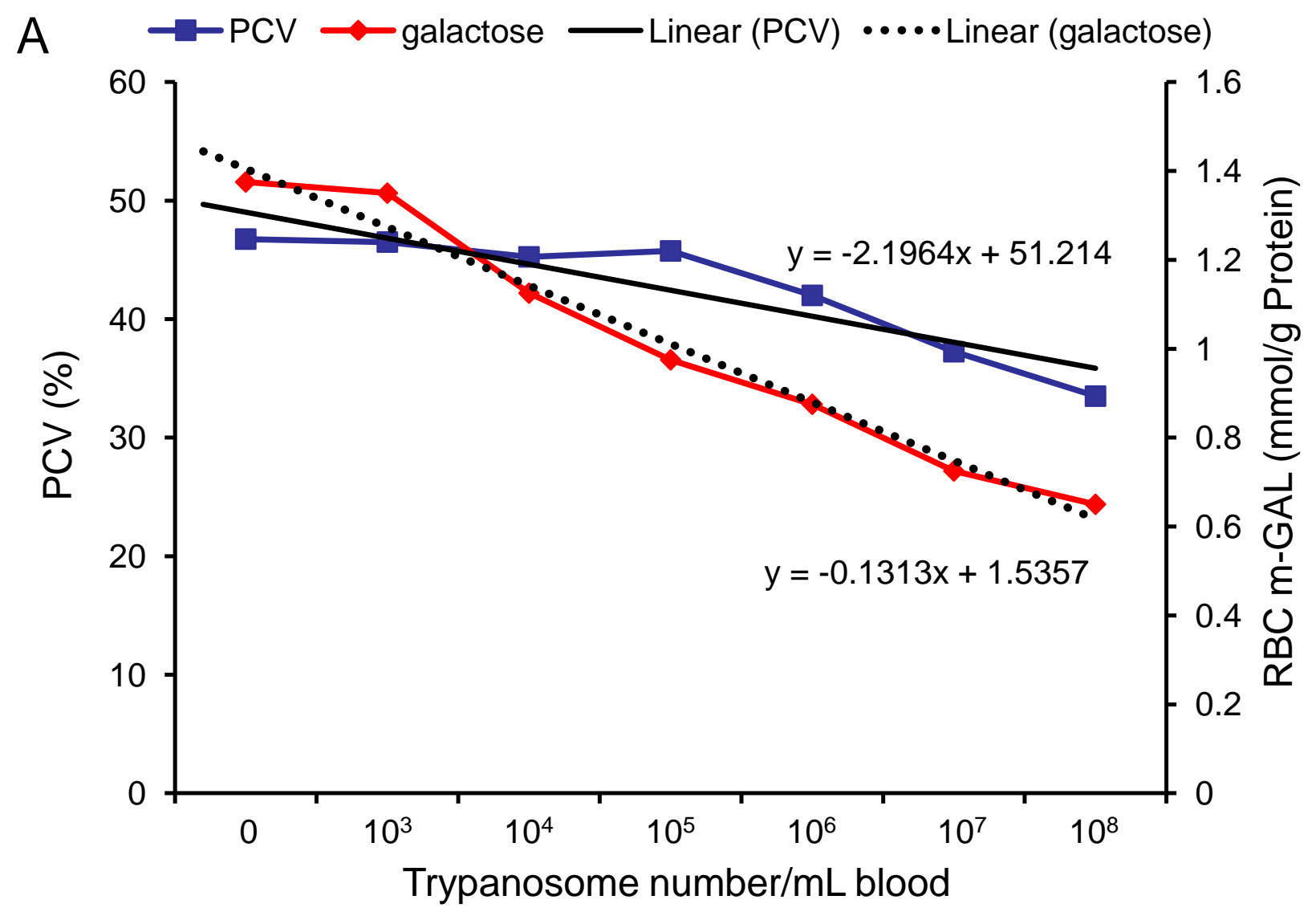

B $\quad$ PCV $\multimap$ galactose - Linear (PCV) $\cdots$... Linear (galactose)

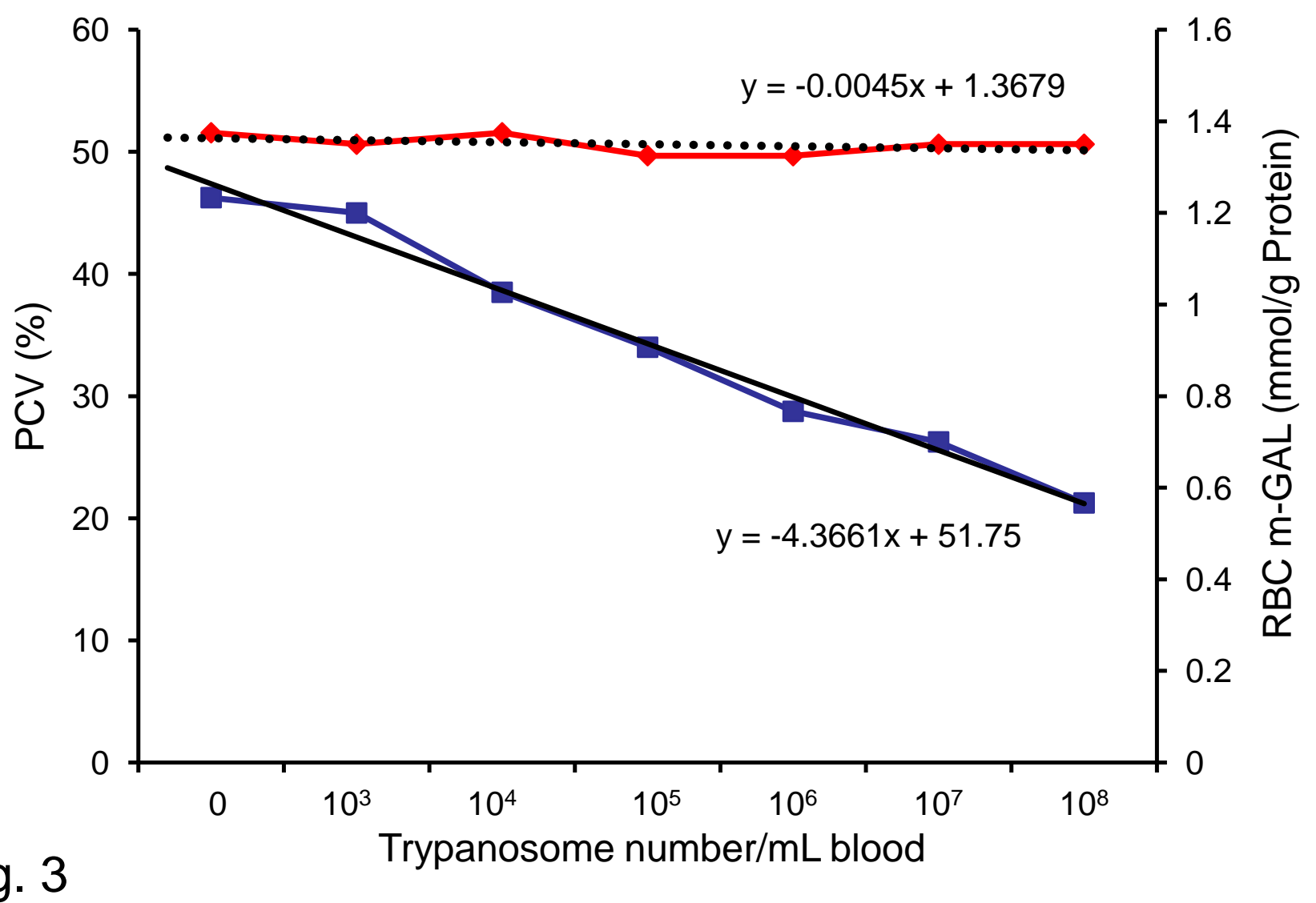

Fig. 3 